\title{
A Bargaining Approach to Power Control in Networks of Autonomous Wireless Entities
}

\author{
Vaggelis G. Douros, George C. Polyzos and Stavros Toumpis \\ Mobile Multimedia Laboratory, Department of Informatics, \\ Athens University of Economics and Business \\ Patision 76, 10434, Athens, Greece \\ \{douros, polyzos, toumpis\}@aueb.gr
}

\begin{abstract}
Interference is a fundamental problem in wireless networks, even in sparse topologies. Power Control (PC) is a powerful tool for mitigating it, however how to apply it to achieve the desired results is not obvious and satisfying the goals of all entities is not always possible. We introduce the Bargaining Foschini-Miljanic $(B F M)$ algorithm, a (partially) distributed algorithm that combines the well-known Foschini-Miljanic (FM) PC algorithm for wireless networks with bargaining between (unsatisfied) entities, in order to maximize the number of entities that gain access to the medium and to achieve additional goals (such as fairness and improved performance). We compare this scheme with the already proposed policy of simply re-applying the $F M$ algorithm to a smaller setup, imposing on the "weakest" node (the one that is furthest from its SINR target) to "power off". Simulations show that our scheme leads to a larger number of efficient solutions without the need to consistently exclude specific nodes. Moreover, our algorithm improves significantly the fairness of the system.
\end{abstract}

\section{Categories and Subject Descriptors}

C.2.1 [Network Architecture and Design]: Wireless Communication; K.6.2 [Installation Management]: Pricing and Resource Allocation.

\section{General Terms}

Algorithms, Economics, Management, Performance

\section{Keywords}

Fairness, Interference, Signal-to-Interference-plus-Noise-Ratio

\section{INTRODUCTION}

Radio spectrum is both a limited and (often) underutilized resource [1]. This makes interference and interference mitigation critically important for wireless networks.

Applying transmitter power control (i.e., a smart selection of the transmission powers so as to achieve high performance) is a

Permission to make digital or hard copies of all or part of this work for personal or classroom use is granted without fee provided that copies are not made or distributed for profit or commercial advantage and that copies bear this notice and the full citation on the first page. To copy otherwise, or republish, to post on servers or to redistribute to lists, requires prior specific permission and/or a fee.

MobiWac'10, October 17-18, 2010, Bodrum, Turkey.

Copyright 2010 ACM 978-1-4503-0277-7/10/10...\$10.00. classical and widely adopted practice to mitigate the interference and can increase the network capacity significantly.

Our model considers a wireless network as a collection of directly interfering links. Wireless (multichannel) cellular networks, as well as wireless ad hoc networks and Wireless Local Area Networks (WLANs) are examples that belong to this broad definition of the term "wireless network". Under this setting, all entities $^{\dagger}$ have a predefined performance target, typically related to their Signal-to-Interference-plus-Noise-Ratio (SINR). Whereas our model is valid even in the traditional cellular setup, we are mostly interested in more challenging cases where entities belong to different operators and have different targets. For example, we consider neighboring WLAN Access Points that serve their own clients.

By default, centralized solutions cannot be applied. Furthermore, links are competitors as each one tries to maximize its target and has no incentive to help other entities to achieve their targets. For these SINR-based scenarios, the application of the famous Foschini-Miljanic (FM) power control algorithm [5] may lead to the satisfaction of SINR targets for all entities. However, there are many cases where $F M$ power control is not sufficient as some entities do not reach their SINR targets.

Figure 1 shows the results from the application of the FM algorithm into three small wireless network setups consisting of 4 , 7 and 10 links (pairs of transmitters/ receivers). For each setup, 50000 different scenarios were simulated where uniformly distributed links apply the $F M$ algorithm to achieve their SINR targets. The values of the various parameters of each experiment are presented in Table 1. Figure 1 presents the number of topologies that led to either i) a feasible solution (all links reached their SINR targets), or ii) an infeasible solution (at least one link did not). As it is shown even in these small setups where few entities coexist (which of course are not a representative for each possible setup), FM scheme leads to infeasible solutions for a significant number of cases (over $10 \%$, over $30 \%$, and over $60 \%$, for 4, 7, and 10 links respectively). To combat these undesirable situations, we present the Bargaining Foschini-Miljanic (BFM) algorithm, a (partially) distributed algorithm that combines the powerful $F M$ scheme with a bargaining rule with a view to maximizing the number of entities that achieve their SINR targets. The algorithm works on top of $F M$ for those scenarios that $F M$ failed to find an operating point that satisfies the targets of all entities. For these cases, a pair of transmitters that have not

\footnotetext{
† The terms "link", "node", "entity" and "transmitter" all refer to the entity that applies the power control algorithm.
} 
achieved their SINR targets is (consecutively) selected and they start negotiations where one transmitter offers some reward to the other, asking it in turn for a reduction to its transmission power.

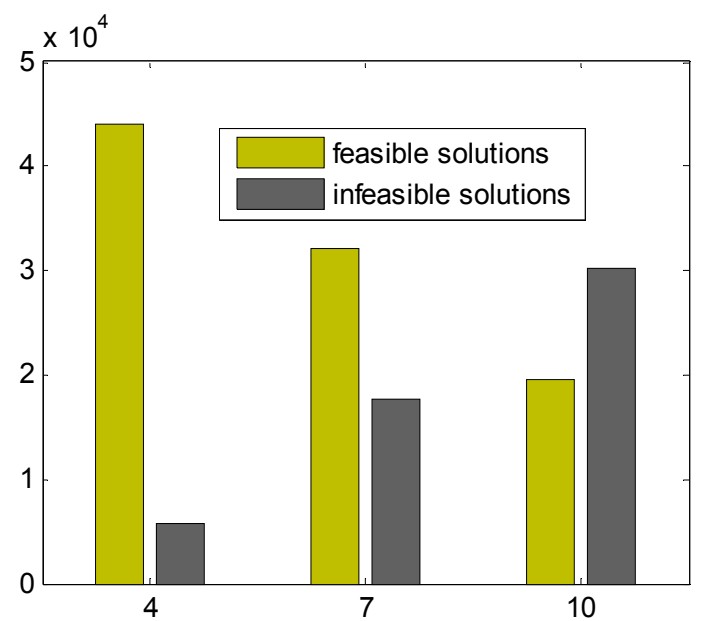

Figure 1: Application of the Foschini-Miljanic (FM) algorithm. The horizontal axis depicts the number of the links of the topology and the vertical axis the number of experiments.

Simulations show that $B F M$ is able to find a large number of cases where the maximum possible numbers of entities achieve their targets. This is quite promising as it is a result of multiple mutual agreements of autonomous entities. This is not the case for the already proposed "cell removal" approach [11], in which the "weakest" entity (the one that is furthest from its target) is enforced to "power off" and then the standard FM power control algorithm is applied to that smaller setup. This policy is quite difficult to implement in practice, as autonomous entities are not obliged to power on/ off based on the instructions of an external referee. Moreover, if $B F M$ is applied many times for the same set of links, the fairness of the system will be improved significantly. This desired property is a consequence of the bargaining among the unsatisfied nodes: In their following attempts to transmit, they have better chances to achieve their targets, as they may use their accumulated rewards to increase the reward they offer during the negotiations. On the other hand, the "cell removal" idea is by design unfair, as the weakest node is always obliged to power off.

To sum up, the key contributions of $B F M$ are threefold: Firstly, $B F M$ maximizes the number of satisfied nodes for a number a cases that $F M$ fails to find a desirable operating point (and such cases exist even in sparse topologies). Secondly, it dramatically increases the fairness of the system, as the set of unsatisfied nodes varies with the time. Last, but not least, the above two properties are achieved without needing to impose a predefined policy that might not be appealing to these (by definition) autonomous entities. Consequently, the bargaining process provides the right incentives to the (unsatisfied) nodes so as to agree with each other on who (and at which transmission power level) will transmit.

\section{Review of Related Work}

Consider a setting in which a number of links, all susceptible to thermal noise, share a common channel. An important question is the following: Can we find a "target" power vector $\boldsymbol{P}^{\boldsymbol{t}}$ so that $\operatorname{SINR}_{i}(k) \geq \gamma_{i}^{t}$ for each link $i$ ? In [5], Foschini and Miljanic were the first who answered positively the question: Given the common $\gamma^{t}$ for each link and provided that a "genie" informs us that there is a feasible unknown power vector $\boldsymbol{P}^{t}$ that achieves this SINR target, can we find it in a fully distributed way? Their work was subsequently simplified by Bambos [4], who showed the following equivalent form of the Foschini-Miljanic formula that is applied by each link during every round $k$ of the algorithm,

$$
P_{i}(k+1)=\gamma_{i}^{t} \frac{P_{i}(k)}{\operatorname{SINR}_{i}(k)}
$$

which we refer to as the simplified Foschini-Miljanic algorithm. This algorithm is fully distributed, as there is no need for communication (and cooperation) among links. If all links apply this algorithm, it is guaranteed that they will achieve their targets after a number of rounds (if this is feasible).

In [8], Mitra showed that this power vector $\boldsymbol{P}^{t}$ is Pareto optimal, in the sense that any power vector $\boldsymbol{P}$ that satisfies the SINR target for each link demands at least as much power for every transmitter and at least one transmitter's power to be greater. I.e., $P \geq P^{t} \quad$ component-wise. Furthermore, he proposed an asynchronous (i.e., all links do not necessarily have to update their transmission power concurrently) version of the FoschiniMiljanic algorithm. In [6], Grandhi, Zander and Yates incorporated a $P_{\max }$ constraint for each link and restate the simplified Foschini-Miljanic as:

$$
P_{i}(k+1)=\min \left\{P_{\max }, \gamma_{i}^{t} \frac{P_{i}(k)}{\operatorname{SINR}_{i}(k)}\right\} .
$$

We can easily see that this algorithm converges to a state where each transmitter has either achieved its SINR target $\left(\gamma_{i}^{t}=\operatorname{SINR}_{i}(k)\right)$ or it is below its target and transmits with $P_{\max }$.

As a final note, as in our case no entity can dictate the power levels, but transmitters can set their transmission powers independently, a game theoretic treatment seems more natural, with the various entities competing among themselves. In [10], Xiao, Shroff and Chong propose the idea of soft SINR targets. This means that links adapt their SINR targets to the channel conditions (as a link feels more interference, it decreases its SINR target). Pricing of the transmission power is used as a mechanism to encourage the links to adjust their targets, achieving some sort of admission control. Thus, the number of links that achieve their SINR targets is increased compared to the $F M$ scheme (for the infeasible cases). Our scheme avoids introducing this punishment mechanism. Moreover, we focus on a solution that is based entirely on the links' desire to negotiate, keeping their SINR targets invariable. In [7], Leung and Sung show that the simplified Foschini-Miljanic scheme is equivalent to the following utility function $U_{i}=-\left|\gamma_{i}^{*}-\operatorname{SINR}_{i}(k)\right|$ that each transmitter tries to maximize. However, nothing new is proposed for the cases where SINR targets are not feasible for all transmitters. Other classical utility and cost functions that are extensively used in noncooperative power control algorithms are discussed in [2] and [9]. However, they do not focus on maximizing the number of feasible links, but they incorporate pricing as a method to find a (more efficient) Nash Equilibrium. 


\section{The Bargaining Foschini-Miljanic Algorithm (BFM)}

In this section, we shall provide an overview of the Bargaining Foschini-Miljanic Algorithm (BFM). As we mentioned, BFM works on top of $F M$ for the cases that $F M$ does not find a feasible solution for all $N$ links of the topology. This is expressed as $\left|P_{i}(k+1)-P_{i}(k)\right| \leq \varepsilon, \forall i$ and $\exists \mathrm{i}: \operatorname{SINR}_{\mathrm{i}}(\mathrm{k})<\gamma_{\mathrm{i}}^{\mathrm{t}}$, where $\varepsilon$ is a small positive quantity. As we discussed in the previous section, $F M$ is unable to help the unsatisfied nodes so as to reach their SINR targets. Given the fact that $F M$ is an algorithm that can always find a feasible solution (if a solution exists), it is impossible for all links to achieve their targets.

$B F M$ tries to maximize the number of transmitters $(N-q)$ that will be able to transmit at the desired SINR, i.e., $\max _{1 \leq q \leq N}\{N-q\}$ s.t. $\operatorname{SINR}_{\mathrm{q}}(k) \geq \gamma_{q}^{t}$. More specifically, we focus on finding a feasible solution just for $N-1$ links. We call this state a "(N-1)-feasible" solution to distinguish it from the standard feasible solution where $N$ links achieve their targets. This is a reasonable choice for many practical cases, where few nodes coexist. However, our algorithm may be extended so as to find the maximum subset of transmitters $(N-q)$ that satisfy their targets. Consequently, in the state that stopped the $F M$ algorithm, transmitters that have achieved their targets have no incentive to change their powers. So, they are not interested in participating in any negotiation, as they are satisfied with the current "status quo". On the other hand, a transmitter that is below its target would definitely like to convince one or more transmitters to reduce its/ their transmission power(s) so as to improve its SINR, as the interference that it perceives would decrease. For this reason, we introduce another parameter, the available Budget $B$ of each transmitter that can be used for negotiations among transmitters. The Budget could be based on real or virtual currency.

$B F M$ works as follows: Firstly, among the transmitters that are below the SINR target, a pair of them is selected. The choice of this pair is random, as it is not based on some predefined rule. However, this process implies that there is a sort of communication among the transmitters (adding also a transmission overhead of the order of $N$ per transmission round), as they should announce if they are "satisfied" or "unsatisfied" nodes. This exchange of messages can be easily realized through the reverse link, similarly to [11]. Of course, this means that $B F M$ is a partially distributed algorithm. We shall discuss later how we could implement a fully distributed algorithm, reducing this overhead too. The first transmitter, let's say $T x_{1}$, is the one that will make an offer to the second transmitter, $T x_{2}$. This offer, $R_{1->2}$, expresses the amount of the Budget (i.e., the reward) that $T x_{1}$ is willing to offer:

$$
R_{1->2}=\max \left\{0, B_{1}(k) \frac{\operatorname{SINR}_{1}(k)}{\gamma_{1}^{t}(k)} \mathrm{P}_{r e d}\right\} .
$$

$R_{1->2}$ is proportional to its current Budget, inversely proportional to its percentage distance from its SINR target and proportional to the percentage reduction $\mathrm{P}_{\text {red }}$ that it will ask for. $\mathrm{P}_{\text {red }}$ is

\footnotetext{
${ }^{\ddagger}$ We assume that transmission powers may take any value in $\left[0, P_{\max }\right]$.
}

computed in (4)-(7). The idea is the following: $T x_{1}$ has an incentive to offer some reward to $T x_{2}$ provided that the latter may reduce its power to a level that will allow $T x_{1}$ to reach its target in the following round of BFM. For this reason, $T x_{1}$ computes the maximum acceptable interference $\operatorname{INTERF}_{O P T}(k)$ that it should perceive so as to achieve its SINR target, given its current transmission power at round $k$ :

$$
\gamma_{1}^{t}=\frac{G_{11} P_{1}(k)}{I N T E R F_{O P T}(k)} .
$$

Moreover, using the information collected from the reverse link, $T x_{1}$ estimates the level of interference from $T x_{2}$. This, in general, demands a unicast exchange of messages between $T x_{1}$ and $T x_{2}$, so that the latter reveals its current transmission power. However, by using the fact that when $F M$ fails to find a feasible solution, all transmitters below their targets transmit at $P_{\max }$, this exchange is not necessary-at least for the first round of $B F M$.

$$
\begin{aligned}
& \operatorname{SINR}_{1}(k)=\frac{G_{11} P_{1}(k)}{\sum_{j \neq 1} G_{j 1} P_{j}(k)+n}=\frac{G_{11} P_{1}(k)}{\sum_{j \neq 1,2} G_{j 1} P_{j}(k)+G_{21} P_{2}(k)+n} \\
& \Leftrightarrow \operatorname{SINR}_{1}(k)=\frac{G_{11} P_{1}(k)}{I N T E R F_{j \neq 1,2}(k)+G_{21} P_{2}(k)}
\end{aligned}
$$

After that, $B F M$ compares the $\operatorname{INTERF}_{O P T}(k)$ with the interference $I N T E R F_{j \neq 1,2}(k)$ that receives from all other sources except of $T x_{2}$ :

$$
\begin{aligned}
& \operatorname{INTERF}_{j \neq 1,2}(k) \leq \operatorname{INTERF}_{O P T}(k) \Rightarrow \\
& G_{21} P_{2 O P T}=I N T E R F_{O P T}(k)-\operatorname{INTERF}_{j \neq 1,2}(k)
\end{aligned}
$$

and estimates which is the minimum $\mathrm{P}_{r e d}$ that it can ask for:

$$
\mathrm{P}_{\text {red }}=\max \left\{0, \frac{P_{2 O P T}}{P_{2}(k)}\right\} \text {. }
$$

Of course, in the case that even if $T x_{2}$ powers off, $T x_{1}$ cannot achieve its target with its current transmission power, then it simply offers no reward to $T x_{2}$.Up to now, we showed how a transmitter defines its reward, as well as the percentage reduction of the transmission power that it demands. From the other side, if the reward is non-zero, $T x_{2}$ has to decide whether to accept it or not. For this reason, $T x_{2}$ computes the quantity

$$
R_{2->1}=\max \left\{0, B_{2}(k) \frac{\operatorname{SINR}_{2}(k)}{\gamma_{2}^{t}(k)} \mathrm{P}_{r e d}\right\},
$$

that expresses the reward that it would have given if it had asked for the same percentage reduction $\mathrm{P}_{\text {red }}$. If $R_{2->1} \leq R_{1->2}$, then $T x_{2}$ accepts the reward, and transmits at the agreeing transmission level: 


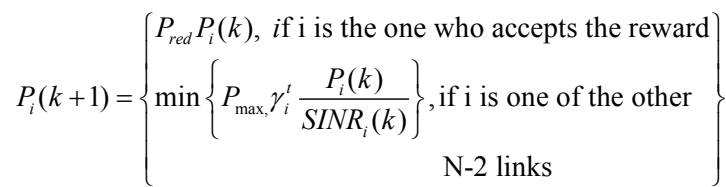

This permits $T x_{1}$ to achieve its target. In addition, the other $\mathrm{N}-2$ transmitters (including the unsatisfied ones) may use $F M$ to improve their SINRs as the interference that they feel is smaller due to the decrease of the transmission power of $T x_{2}$. In the case that $T x_{2}$ rejects the offer, and given the fact that $F M$ had stopped in an infeasible state where power levels cannot change anymore, $T x_{1}$ voluntarily decides to decrease by a small amount its transmission power $(c \%)$ so that the interference that the other transmitters perceive will change. So, the other $N-1$ transmitters could be able to apply $F M$ again:

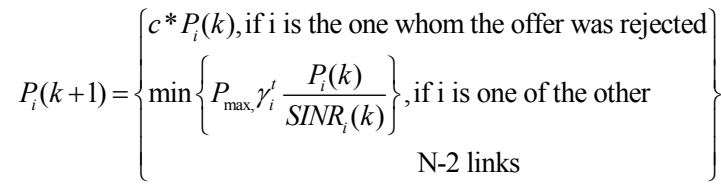

It is worth mentioning that, though this voluntary reduction of the transmission power seems a bad choice, it may be proven beneficial. Otherwise, all transmission powers would remain at the same level. This slight reduction may change the set of unsatisfied nodes and may give to $T x_{1}$ another opportunity (in a following round of $B F M$ ) to offer a different reward to another transmitter.

A key advantage of $B F M$ is that through the negotiation, $N-1$ nodes will apply the $F M$ algorithm whereas one node will decrease its power. This update scheme assures that there will be some evolution of the powers of the transmitters, which would have never been possible if all of them had applied $F M$ (if, at some time, $N$ links applied $F M$, they would end up-in a few iterations-again to the last state of $F M$ without being able to change their transmission powers). Moreover, transmitters that have achieved their targets are not in danger to fall below them, as the unsatisfied nodes try to achieve their powers only by trying to reduce the interference that they perceive (and not by trying to increase their power at a level above the one specified by the $F M$ algorithm). If, after this bargaining process, $N-1$ links achieved their SINR targets, then BFM stops. Otherwise, another pair among the (probably) new set of unsatisfied transmitters is selected and a new negotiation takes place. We point out that the set of unsatisfied transmitters may change (actually, it may be reduced), if a link achieves its SINR target during this bargaining.

We mention that, contrary to $F M$ (where a transmitter either has achieved exactly its SINR target or transmits at $P_{\max }$ ), $B F M$ may lead to a case where some transmitters have exceeded their SINR targets. In other words, $B F M$ stops at the first operating point that satisfies the targets of $N-1$ links. It is an open issue whether this negotiation process (if it goes on) may lead to even better operating points, e.g. in the sense of finding the component-wise minimum power vector that satisfies the targets of $N-1$ links. As a final note, we point out that, because after each negotiation step one transmitter decreases its power, all other transmitters may improve their SINR. This means that bargaining causes a positive externality [9] not only to the transmitter: i) that either rejects the offer or ii) whom the offer is accepted, but also to all $N-2$ entities of the topology that did not participate in this negotiation. However, keep in mind that even if a negotiation leads to an agreement and one node reduces its transmission power so that the other node achieves its target, this does not in general mean that all other unsatisfied nodes will achieve their targets. They may improve their SINR but not to that level so as to

To sum up, Diagram 1 depicts the process that $\mathrm{Tx}_{1}$ and $\mathrm{Tx}_{2}$ follow to agree to the level of the powers at which they will transmit.

1) $\mathrm{Tx}_{1}$ computes through (4) its maximum acceptable interference power $\operatorname{INTERF}_{O P T}(k)$.

2) $\mathrm{Tx}_{1}$ sends to $\mathrm{Tx}_{2}$ a unicast message asking for its power. $\mathrm{Tx}_{2}$ informs $\mathrm{Tx}_{1}$ through a unicast message for its transmission power (sometimes, this step is optional).

3) $\mathrm{Tx}_{1}$ computes through (5)-(7) $\mathrm{P}_{\text {red }}$ : the percentage reduction of the power of $\mathrm{Tx}_{2}$ that is needed so that $\mathrm{Tx}_{1}$ achieves its target.

4) $\mathrm{Tx}_{1}$ computes through (3) the Reward $R_{1->2}$ that is willing to offer to $\mathrm{Tx}_{2}$.

5) $\mathrm{Tx}_{2}$ computes through (8) the Reward $R_{2-1}$ that would have given if $\mathrm{Tx}_{2}$ had asked for the same $\mathrm{P}_{\text {red }}$.

6) If $R_{2->1} \leq R_{1->2}, \mathrm{Tx}_{2}$ accepts the offer and transmits at $\mathrm{P}_{\text {red }} * P_{i}(k)$. Otherwise, $\mathrm{Tx}_{2}$ updates its power using the $F M$ algorithm.

7) If $\mathrm{Tx}_{2}$ accepts its offer, then $\mathrm{Tx}_{1}$ updates its power according to the FM scheme and achieves its target. Otherwise, $\mathrm{Tx}_{1}$ reduces $c \%$ its current power.

Diagram 1: Steps that $\mathrm{Tx}_{1}$ (the transmitter who makes an offer) and $\mathrm{Tx}_{2}$ (the transmitter who receives an offer) follow to decide their new powers.

\section{4. (Trunc) FM and BFM in Practice}

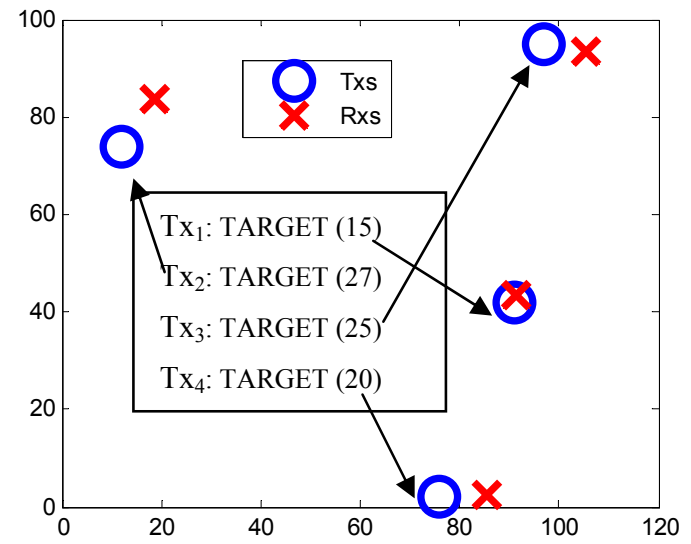

Figure 2: A small wireless network setup consisting of 4 links. In that particular case circles transmit whereas crosses receive. Each $T x$ wants to communicate with its closest $R x$, but it causes interference to all other links. 
In this section, we shall provide a small example that depicts how (and why) the $F M$ and the $B F M$ algorithms work. Figure 2 presents a small wireless network topology consisting of 4 links that coexist (and share the same channel). This setup may correspond either to a cellular network, where the Txs are Base Stations and the Rxs are Mobile Nodes, or to a wireless ad hoc network, where the Txs are the nodes that (in that particular case) transmit data, whereas the Rxs are the nodes that receive data.

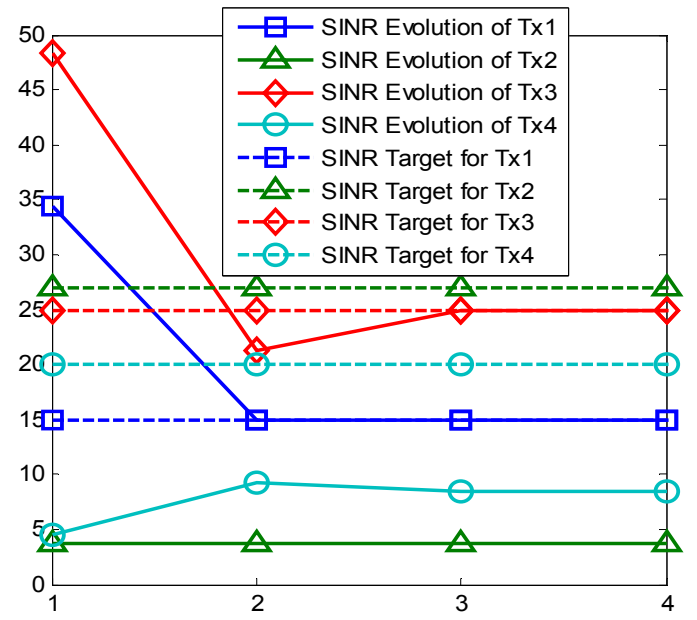

Figure 3: SINR evolution of links after application of $F M$. Horizontal dashed lines correspond to SINR targets of BSs. After 4 iterations, $F M$ stops and has led to an infeasible solution as both $T x_{2}$ and $T x_{4}$ are below their SINR targets.

Figure 3 presents the SINR evolution after the application of the $F M$ power control algorithm. After 4 iterations, $F M$ stops at an infeasible state, as both $\mathrm{Tx}_{2}$ and $\mathrm{Tx}_{4}$ are below their SINR targets (which are presented as dashed lines on the diagram). So, the new target is to find a scheme that leads to power levels so that 3 (out of 4) links achieve their SINR targets.

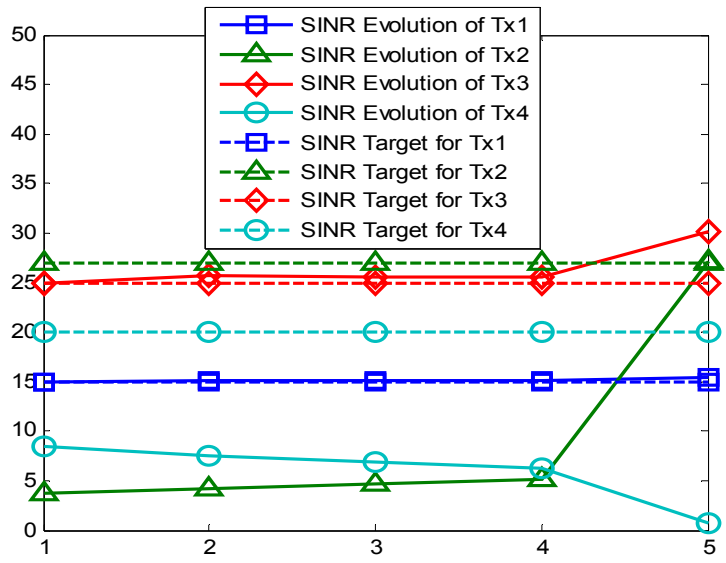

Figure 4: SINR evolution of BSs after application of $B F M$. Initial SINRs are the ones of the last round of $F M$. Horizontal dashed lines correspond to the SINR targets of links. After 5 iterations, $B F M$ stops and has led to a "(N-1)-feasible" solution as only $\mathbf{T x}_{\mathbf{4}}$ is below its SINR target.

We firstly apply the $B F M$ power control algorithm, which takes as input the last state of $F M$ (Figure 4). This is the reason why the
SINR of each link at the $1^{\text {st }}$ round of $B F M$ coincides with the SINR value at the last $\left(4^{\text {th }}\right)$ round of $F M$. As we can notice, $B F M$ finds a "(N-1)-feasible" solution at its $5^{\text {th }}$ iteration, in which 3 out of 4 links have achieved their SINR targets. It is worth describing how the algorithm leads to that result. During the $1^{\text {st }}$ iteration, $\mathrm{Tx}_{2}$ and $\mathrm{Tx}_{4}$ were below their SINR targets. So, one of them $\left(\mathrm{Tx}_{4}\right)$ was randomly selected to make on offer to the other $\left(\mathrm{Tx}_{2}\right)$. This offer was rejected as, during the $2^{\text {nd }}$ iteration, $\mathrm{Tx}_{4}$ reduced a bit (in our example, 10\%) its transmission power (Figure 5). So, $\mathrm{Tx}_{2}$ improved a bit its SINR. If $\mathrm{Tx}_{2}$ had accepted the offer, then $\mathrm{Tx}_{4}$ would have achieved its SINR target (and $\mathrm{Tx}_{2}$ would have decreased its power). The same situation arose again during the $2^{\text {nd }}$ and $3^{\text {rd }}$ iteration of $B F M$ : $\mathrm{BS}_{4}$ offered some reward and $\mathrm{Tx}_{2}$ refused so the former reduced its transmission power (at $90 \%$ of its previous power level) and the latter improved its SINR. However, at the $4^{\text {th }}$ iteration, roles changed and $\mathrm{Tx}_{2}$ was selected to offer some reward to $\mathrm{Tx}_{4}$. This negotiation was successful as $\mathrm{Tx}_{4}$ accepted the reward and reduced its power to that level so that $\mathrm{Tx}_{2}$ achieved its target at the $5^{\text {th }}$ iteration. In addition, this significant reduction of the transmission power of $\mathrm{Tx}_{4}$ was also (somehow) beneficial for $\mathrm{Tx}_{1}$ and (mainly) $\mathrm{Tx}_{3}$ as they managed to improve their SINR (a bit) above their SINR targets.

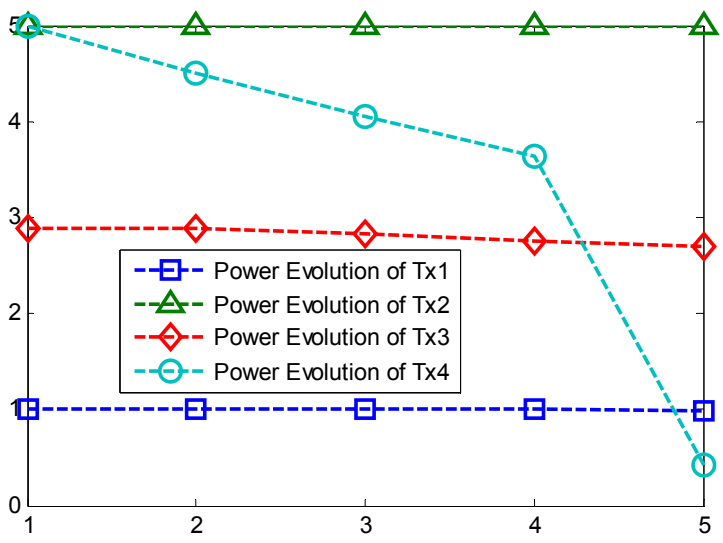

Figure 5: Power evolution of BSs after application of $B F M$. Initial Powers are the ones of the last round of $F M$. At the $1^{\text {st }}$, $2^{\text {nd }}$ and $3^{\text {rd }}$ rounds of $B F M, T x_{4}$ offers some reward to $\mathrm{Tx}_{2}$. As $T x_{2}$ always refuses, $T x_{4}$ slowly reduces its power. At the $4^{\text {th }}$ round, $\mathrm{BS}_{2}$ offers some reward and $\mathrm{Tx}_{4}$ accepts it. So, $\mathrm{Tx}_{2}$ achieves its SINR target at the $5^{\text {th }}$ round.

Moreover, we compare $B F M$ with the proposed approach of demanding from the weakest BS (the one that is furthest from its SINR target) to power off [11]. We call this approach the Trunc $F M$ power control algorithm, as $N-1$ links update their powers according to $F M$, whereas one link powers off. This algorithm is (also) partially distributed, as cooperation among links is necessary to find out the weakest one.

Figure 6 shows the SINR evolution after the application of Trunc $F M$. At first, we notice that $\mathrm{Tx}_{2}$ is the one who powers off. This is due to the fact that, at the last iteration of $F M, \mathrm{Tx}_{2}$ was further from its target than $\mathrm{BS}_{4}$. As $\mathrm{BS}_{2}$ powers off, the SINRs of all other links are improved (to see this, just compare the SINR values at the $1^{\text {st }}$ round of Trunc FM and the $5^{\text {th }}$ round of $F M$ ). However, after just 2 more iterations, Trunc FM stops (as transmission powers at $2^{\text {nd }}$ and $3^{\text {rd }}$ round remained invariable- 
Figure 7). In fact, this state is infeasible, as, although $\mathrm{Tx}_{2}$ powers off, $\mathrm{Tx}_{4}$ remains below its SINR target. In other words, Trunc FM failed to find an operating point where 3 links achieve their SINR targets, whereas $B F M$ managed to find a solution.

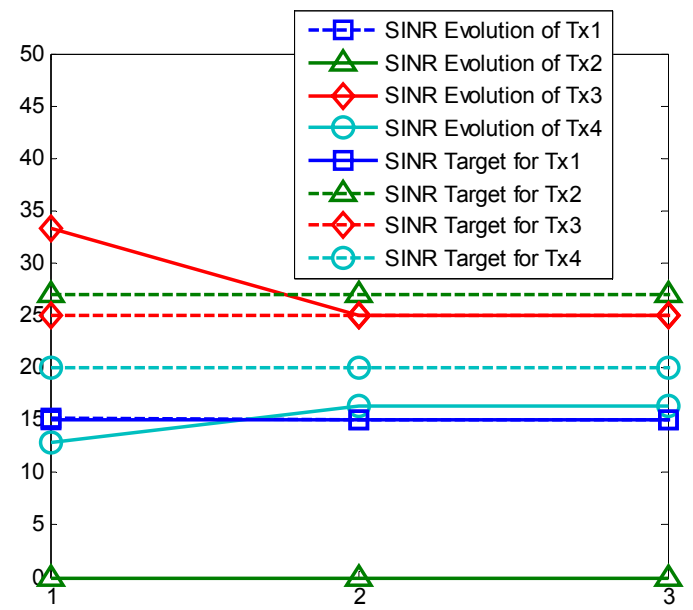

Figure 6: SINR evolution of links after application of Trunc $F M . ~ T x_{2}$ powers off, as it was the furthest away from its target (round 5, Figure 3). $T \mathbf{x}_{4}$ cannot achieve its target and the algorithm stops at the $3^{\text {rd }}$ round leading to an infeasible state.

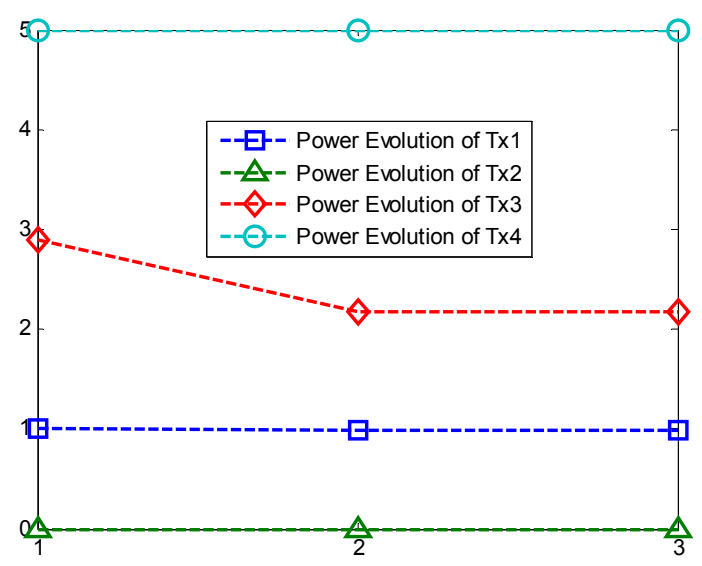

Figure 7: Power evolution of links after application of Trunc $F M$. Tx $x_{2}$ powers off, as it was the weakest node. Trunc FM stops at the $3^{\text {rd }}$ round, as there is no difference between power levels at the $2^{\text {nd }}$ and $3^{\text {rd }}$ rounds.

This small example illustrates how powerful the integration of power control and bargaining can be. Though Trunc FM imposed to a BS to power off, its choice was finally wrong, as the other BS did not achieve its target. On the other hand, the mutual negotiations between these links were more effective as they led to a "(N-1)-feasible" solution.

\section{Performance Evaluation}

\subsection{BFM: Extra “(N-1)-feasible” Solutions}

In this subsection, we evaluate the performance of the BFM algorithm through simulations. Simulation parameters are presented in Table 1. We already examined (through Figure 1) how often $F M$ leads to an infeasible state (even for small setups where few links coexist). Consequently, our focus is limited only to these cases, as for the feasible ones there is no need to apply $B F M$. However, even for those infeasible states where $N$ links cannot achieve their targets, there are some cases where $F M$ has stopped to a " $(N-1)$-feasible" solution finding an operating point where $N-1$ links have already achieved their targets-so there is no need to apply $B F M$. Figure 8 presents the total number of feasible solutions, where feasible includes both the standard solutions $(N$ links achieve the targets) and the " $(N-1)$-feasible" solutions. As we can see, from the 50000 different scenarios of each topology, nearly all (when the topology consists of 4 or 7 links) scenarios and over $80 \%$ of scenarios with 10 links led to a feasible solution. However, the most important notice is that a non trivial part of "(N-1)-feasible" solutions (around 7\%, 20\% and 30\% of the total feasible solutions for 4, 7 and 10 links respectively) cannot be identified without the use of BFM! These extra "(N-1)-feasible" solutions correspond to the grey color of each bar of Figure 8 .

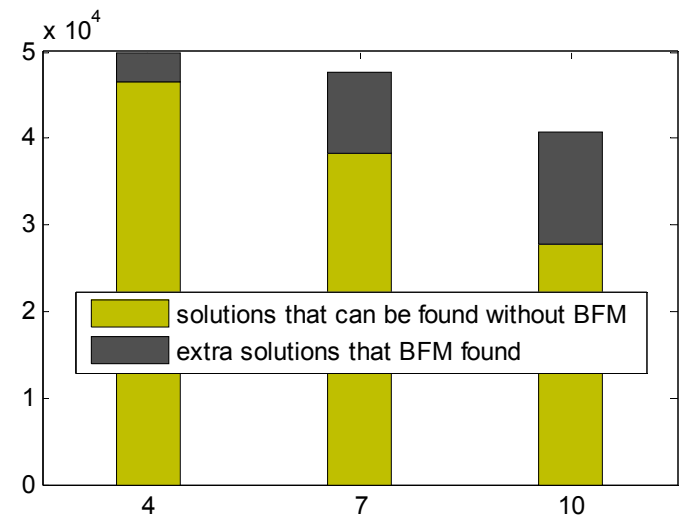

Figure 8: Figure presents the total number of feasible solutions, i.e., cases where either $N$ or $N-1$ links achieved their targets. The grey part of the bars depicts the " $(N-1)$-feasible" solutions that would not have been found without $B F M$.

Table 1: Simulation Parameters

\begin{tabular}{|c|c|}
\hline Parameter & Value \\
\hline \# Links of each Topology & $4,7,10$ \\
\hline \# Scenarios per Topology & 50000 \\
\hline $\begin{array}{c}\text { Max \# Iterations per } \\
\text { Algorithm }\end{array}$ & 1000 \\
\hline Simulation Terrain & A square of size 100 \\
\hline Transmitters (Tx) Distribution & Uniform \\
\hline Receivers ( $\mathrm{Rx}$ ) Distribution & $\begin{array}{l}\mathrm{Rx} \text { is placed randomly in the } \\
\text { interior of a circle of radius } 5 \\
\text { from its associated Tx }\end{array}$ \\
\hline Path Loss Model & $\begin{array}{c}G=f\left(d^{-4}\right), \mathrm{d}: \text { distance between } \\
\text { Tx and } \mathrm{Rx}\end{array}$ \\
\hline Mobility Model & Quasi-static model \\
\hline Noise & $10^{-6}$ \\
\hline $\mathbf{P}_{\max }$ & 5.0 \\
\hline SINR Targets (in $d B$ ) & {$[11,15]$} \\
\hline Initial Transmission Powers & Randomly selected at $\left(0, P_{\max }\right]$ \\
\hline Stopping Parameter $\boldsymbol{\varepsilon}$ & $10^{-4}$ \\
\hline $\begin{array}{c}\text { Parameter c (Voluntarily } \\
\text { Power Reduction) }\end{array}$ & 10 \\
\hline Initial Budget $\mathbf{B}$ & Randomly value at $[100,200]$ \\
\hline
\end{tabular}




\subsection{Performance of $B F M$ vs. Trunc FM}

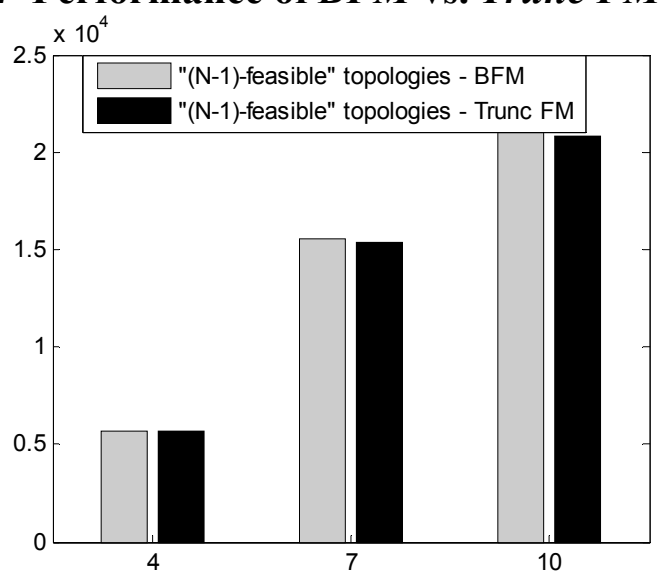

Figure 9: We present the number of "(N-1)-feasible" solutions, after the application of both BFM and Trunc FM for the same scenarios.

Next, we compare the number of " $(N-1)$-feasible" solutions that $B F M$ finds (i.e., the cases where $N-1$ links achieve their SINR targets) with the number of " $(N-1)$-feasible" solutions that Trunc $F M$ finds. As shown in Figure 9, BFM and Trunc FM find more or less the same number of " $(N-1)$-feasible" solutions (actually, BFM outperforms Trunc FM-in these particular scenarios-less than $1 \%$, but this difference is insignificant). However, we stress out that our algorithm presents similar performances with a policy that is inherently unrealistic, as it is very difficult to enforce a link to power off. On the other hand, though $B F M$ expects the nodes to take part in negotiations, they always have the opportunity to deny an offer (or make a zero offer). Moreover, the fact that they present similar performance does not imply that both $B F M$ and Trunc FM always lead to the same state. As we showed in Section 4, there are cases where BFM leads to a " $(N-1)$-feasible" state, whereas Trunc FM leads to an infeasible state (of course, this may happen the other way around too). Furthermore, as we show next, $B F M$ promotes the fairness of the system.

\subsection{BFM vs. Trunc FM: A Note on Fairness}

Another interesting aspect has to do with the following remark: Entities that coexist in the same topology are generally interested in transmitting at various times intervals (transmission rounds). So, independently of whether they achieve their SINR target or not during a particular transmission round, chances are that they will try to transmit again after some time. This implies that a power control scheme should be fair in the sense that, if the same set of entities with the same SINR targets apply long-term the proposed scheme, the set of (un)satisfied entities should vary over time. By definition (see also Section 3), those links that have achieved their SINR targets at the last round of $F M$ will never fall below their targets. Consequently, fairness has to do with whether, trying to find out a "(N-1)-feasible" solution, the unsatisfied node varies with the time (and this will always be one of the unsatisfied nodes at the last round of $F M$ ). We consider a scenario as "unfair", when the node that ends up below its SINR target (i.e., it is "unsatisfied") is always the same.

Unfortunately, Trunc FM is by design unfair, as it always penalizes the weakest node. Consequently, this node will never have the opportunity-not even to try-to transmit, as it will always power off. On the other hand, so as to decide whether $B F M$ is fair, we perform simulations where we focus only on the interesting scenarios, i.e., cases where $N$ links cannot coexist in the same topology and $F M$ did not find a " $(N-1)$-feasible" solution. For these cases, we apply $B F M$ for $M=100$ time intervals (transmission rounds). When $B F M$ is applied for the $(\mathrm{m}+1)^{\text {th }}$ transmission round, links reset their parameters to the last state of the $F M$ algorithm except of their Budgets, which are the ones that arose after the application of $B F M$ at the $\mathrm{m}^{\text {th }}$ transmission round. With this way, we examine whether the rewards that the unsatisfied links may have collected during negotiations of previous transmission rounds will increase their chances to make an offer that will get accepted to a following transmission round (We have already seen that this case may happen during the iterations of the same transmission round).

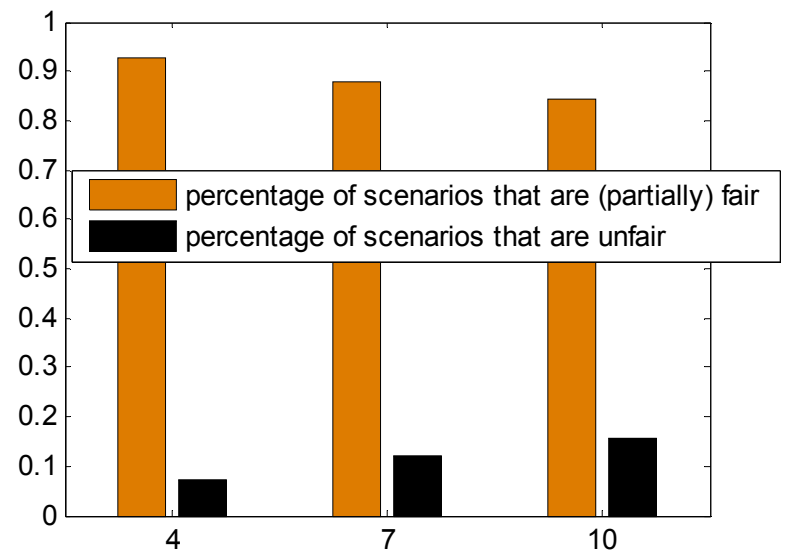

Figure 10: We apply $B F M$ (for 100 transmission rounds) for the same set of nodes, by resetting their parameters to the last state of $F M$. The Budget at the $(\mathrm{m}+1)^{\text {th }}$ round is the one that arises after at the $\mathrm{m}^{\text {th }}$ round. We examine which percentage of scenarios lead to a (partially) fair distribution of the unsatisfied nodes.

Figure 10 shows that $B F M$ increases significantly the fairness of the system, as over $85 \%$ of the scenarios rotate the unsatisfied node, leading to a (partial) fairness. Moreover, we are interested in studying the degree of fairness that $B F M$ can achieve. As in the majority of cases (over $90 \%$ of our studied scenarios), the number of unsatisfied nodes at the last round of $F M$ is 2 (e.g. link , $_{1}$ and $\operatorname{link}_{2}$ ), we focus on that case. A suitable fairness metric expresses the ratio $R=$ (transmission rounds that link $k_{1}$ achieves its target)/(transmission rounds that link $k_{2}$ achieves its target). With this definition, our simulations for 4, 7 and 10 links show that the average fairness that is achieved is (a bit less than) 2:1, in the sense that (on average) in 2 out of 3 transmission rounds one link achieves its SINR target, whereas in 1 out of 3 transmission rounds the other link achieves its SINR target (a related diagram is not included to save space). This result is very promising, being a substantial improvement compared to Trunc FM which always powers off the same link (so, one of these two links would never have the opportunity-not even to try-to transmit!).

\subsection{BFM vs. (Round Robin) Trunc FM: A Note on Efficiency}

In this subsection, we compare BFM with a Round Robin Trunc $F M$ scheme that, instead of always powering off the weakest node, it chooses in a "round robin" way a node among the set of unsatisfied ones to power off. Then, we find among all cases that 
Trunc FM needs to be used are not $N$-feasible, which are the ones where this Round Robin Trunc FM variation will not lead to a "(N-1)-feasible" solution for each node that could power off. In other words, we are interested in cases where, e.g., when node is $_{1}$ chosen to power off, then the other $N-1$ nodes achieve their targets, but when node 2 is chosen to power off, then the other $\mathrm{N}-1$ nodes cannot achieve their targets.

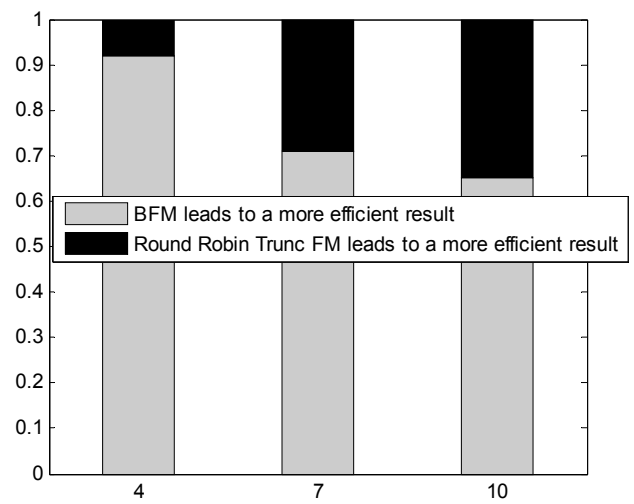

Figure 11: Efficiency of BFM vs. (Round Robin) Trunc FM.

Figure 11 compares the performance of $B F M$ vs. the Round Robin Trunc $F M$ in terms of efficiency. $B F M$ is considered more efficient than the Round Robin Trunc FM (and vice versa), if it leads in more transmission rounds to a state where $N-1$ links achieve their SINR targets. It is shown that $B F M$ is more efficient, a fact which is justified as follows: Through the bargaining process and the mutual agreements between the unsatisfied links, $B F M$ manages to decrease the chances of a "wrong" link lowering its power. A link is characterized as "wrong" if it is chosen by the Round Robin Trunc FM to power off and has as a result that the other $N-1$ links cannot achieve their targets.

\section{Conclusions and Future Directions}

This paper discusses an integration of the classic FoschiniMiljanic power control algorithm with bargaining that may apply to general settings of wireless networks where entities have different targets which cannot be concurrently satisfied for all of them. These cases arises quite often even in small topologies with few entities. Our Bargaining Foschini-Miljanic (BFM) scheme finds a feasible solution in many of these challenging cases, through negotiations between a pair of unsatisfied nodes, where the former offers to the latter some reward with a view to decreasing its transmission power so that the former achieves its target. Through a series of simulations, we compared BFM with the current "status quo", the-so called-Trunc FM approach, where the weakest node enforced to power off. $B F M$ finds an equal number of " $(N-1)$-feasible" solutions with Trunc FM, being more fair (as the set of unsatisfied nodes of a particular scenario varies when the algorithm is applied for many transmission rounds) and efficient (compared e.g. to a case where a Round Robin Trunc FM scheme is applied).Our future plans include the following. This is very important to deal with dense topologies where more links would not achieve their targets. In a different direction, we are interested in extending $B F M$ to be a fully distributed algorithm. This could be done providing that each node selects autonomously whether it will make an offer (and to which node). This will significantly reduce the transmission overhead of our approach. As a further extension, during each round of $B F M$, nodes may even make multiple offers to multiple entities. A discussion on the impact of the budget allocation is also under investigation. It is also challenging to extend our scheme with soft SINR targets, comparing its performance with [10]. Lastly, we are interested in applying our scheme by using discrete power control [3], a challenging topic that has not yet attracted much attention.

\section{REFERENCES}

[1] I.F. Akyildiz, W.Y. Lee, M.C. Vuran and S. Mohanty, "Next generation/ dynamic spectrum access/ cognitive radio wireless networks: a survey," Elsevier Computer Networks, vol. 50, no.13, 2006.

[2] T. Alpcan, T. Başar, R. Srikant and E. Altman, "CDMA uplink power control as a noncooperative game," Wireless Networks (Kluwer), vol. 8, no. 6, November 2002.

[3] E. Altman, K. Avrachenkov, I. Menache, G. Miller, B. Prabhu and A. Shwartz, "Dynamic discrete power control in cellular networks," IEEE Trans. on Automatic Control, vol. 54, no. 10, October 2009.

[4] N. Bambos, "Toward power-sensitive network architectures in wireless communications: concepts, issues, and design aspects," IEEE Personal Communications, vol. 5, no. 3, June 1998.

[5] G.J. Foschini and Z. Miljanic, "A simple distributed autonomous power control algorithm and its convergence," IEEE Trans. on Vehicular Technology, vol. 42, no. 4, November 1993.

[6] S.A. Grandhi, J. Zander and R. Yates, "Constrained power control," Wireless Personal Communications (Kluwer), vol. 1, no. 4, December 1994.

[7] K.K. Leung and C.W. Sung, "An opportunistic power control algorithm for cellular network," IEEE/ACM Trans. on Networking, vol. 14, no. 3, June 2006.

[8] D. Mitra, "An asynchronous distributed algorithm for power control in cellular radio systems," Proc. 4th WINLAB Workshop, 1993.

[9] C.U. Saraydar, N.B. Mandayam and D.J. Goodman, "Efficient power control via pricing in wireless data networks," IEEE Trans. on Communications, vol. 50, no. 2, February 2002.

[10] M. Xiao, N. B. Shroff and E. K. P. Chong, "A utility-based power-control scheme in wireless cellular systems," IEEE/ACM Trans. on Networking, vol. 11, no. 2, April 2003.

[11] J. Zander, "Distributed cochannel interference control in cellular radio systems," IEEE Trans. on Vehicular Technology, vol. 41, no. 3, August 1992. 